\title{
The effects of rivaroxaban, an oral anticoagulant, on human IVD primary cultures
}

\author{
Tezcan Caliskan ${ }^{1}$, Hande Akalan², Ibrahim Yilmaz , Numan Karaarslan4, Duygu Yasar Sirin², \\ Hanefi Ozbek ${ }^{5}$
}

\begin{abstract}
'Department of Neurosurgery, School of Medicine, Namik Kemal University, Tekirdag, Turkey

2Department of Molecular Biology and Genetics, Faculty of Arts and Sciences, Namik Kemal University, Tekirdag, Turkey

${ }^{3}$ Department of Medical Pharmacology, School of Medicine, Istanbul Medipol University, Istanbul, Turkey

${ }^{4}$ Department of Neurosurgery, School of Medicine, Halic University, Istanbul, Turkey ${ }^{5}$ Department of Medical Pharmacology, Bakircay University School of Medicine, Izmir, Turkey
\end{abstract}

Submitted: 5 November 2020; Accepted: 2 May 2021

Online publication: 9 May 2021

Arch Med Sci 2022; 18 (4): 1062-1070

DOI: https://doi.org/10.5114/aoms/136323

Copyright $\odot 2021$ Termedia \& Banach

\section{Abstract}

Introduction: The present study aimed to investigate the potential effects of rivaroxaban, an oral anticoagulant that inhibits the effects of factor $\mathrm{Xa}$, on intact intervertebral disc tissue cells and the extracellular matrix (ECM). Material and methods: Rivaroxaban was applied to primary human cell cultures prepared from tissues of the intervertebral disc. Comparative molecular analyses were performed on non-drug-treated control group samples. Descriptive statistics were presented as the mean \pm standard deviation. An analysis of variance test was performed to determine whether there were significant differences in the mean across the groups. When differences across groups were observed, Tukey's honestly significant difference post-hoc test was used for multiple pairwise comparisons. The significance of the obtained data was determined statistically. The $\alpha$ significance value was $<0.05$.

Results: The cells in the control group and in the rivaroxaban-treated group were viable, healthy, and proliferated $(p<0.05)$. However, the expression levels of the chondroadherin gene (CHAD), cartilage oligo matrix protein (COMP), matrix metalloproteinase (MMP)-13, and MMP-19 genes were changed $(p<0.05)$.

Conclusions: Although rivaroxaban does not suppress cell proliferation due to morphological, biological, and biochemical changes in the intervertebral disc tissue, it may change the expression of genes that are related to ECM maintenance.

Key words: cartilage oligo matrix protein, chondroadherin gene, cytotoxicity, intervertebral disc cells, matrix metalloproteinases, nucleus pulposus, rivaroxaban.

\section{Introduction}

Rivaroxaban belongs to a group of medicines called antithrombotic agents. They are used as an inhibitor of factor $\mathrm{Xa}$, which plays a role in blood clotting [1]. The administration of this novel antithrombotic agent is recommended following the use of anticoagulant drugs, such as warfarin, and it does not require international normalized ratio monitoring [2, 3].

\author{
Corresponding author: \\ Ibrahim Yilmaz PhD \\ Department of \\ Medical Pharmacology \\ School of Medicine \\ Istanbul Medipol \\ University \\ Istanbul, Turkey \\ Phone: +905327012858 \\ E-mail: ibrahimyilmaz77@ \\ yahoo.com
}


The half-life of rivaroxaban is between 7 and $17 \mathrm{~h}$. In addition to its ease of use, it is well absorbed by the gastrointestinal system, its food interaction is minimal, and its bioavailability is $80 \%$. Peak plasma concentrations are attained within approximately $3 \mathrm{~h}$ after oral administration. Thus, it is used for the following: a) to prevent non-valvular atrial fibrillation [4], b) to prevent stroke [5] and blood clots in other veins of the body, c) to treat deep-vein thrombosis and pulmonary embolism, and d) to treat deep-vein thrombosis and the prophylaxis of pulmonary embolism in the lungs [6]. In addition, rivaroxaban is a direct factor Xa inhibitor used once a day for prevention of thrombotic events in patients with atrial fibrillation [7]. The recommended dose is $30 \mathrm{mg} /$ day for a minimum of 21 days. The usage period can be decreased to 12 days for knee replacement surgery, and it can be increased to 35 days for hip replacement surgery. Rivaroxaban-related side effects at $20 \mathrm{mg} /$ day for the prevention of stroke in atrial fibrillation have been reported in the literature. Recorded adverse side effects include gastrointestinal hemorrhage, urogenital hemorrhage, epistaxis, ulorrhagia, vitreous hemorrhage, hematoma, hemoptysis, and skin/subcutaneous hemorrhages. In addition, side effects such as hemorrhage after surgery, limb edema, pain, fever, and orthostatic hypotension or faintness have been reported. Some studies have also reported that fatigue, intracerebral hemorrhage, pseudoaneurysm, or tachycardia may occur in patients using rivaroxaban [8].

However, no previous study has focused on the positive and negative effects of this pharmacological agent on intervertebral disc tissue. For that reason, the effect of this drug, which is frequently used before and after the operation, on the healing process is unknown. This study considering the effects and toxicity of rivaroxaban on intervertebral disc (IVD) primary cultures can shed some light on this subject. Therefore, the findings obtained through the present research could contribute to the literature.

The aim of this study is to investigate the effects of rivaroxaban on primary cell cultures prepared from human intervertebral disc tissue at the pharmaco-molecular level. To achieve this aim, the expression level of chondroadherin (CHAD) [9-14], an NP-specific marker, was examined. The level of the cartilage oligo matrix protein (COMP) [15-18], which plays a role in intervertebral disc catabolic metabolism, was also tested. In addition to these markers, the levels of matrix metalloproteinase (MMP)-13 and MMP-19 [19] were examined. Both MMP-7 and MMP-19 play roles in remodeling the intervertebral disc and the extracellular matrix (ECM) structure, as well as in the catabolic pathways of these tissues.

\section{Material and methods}

The present research was conducted with the approval of the Ethics Committee of University School of Medicine (ethical permission no. 10840098/604.01.01/E.44192, 29). Written informed consent was obtained from all patients prior to their inclusion in the study.

To minimize experimental errors, assays were repeated at least three times. The tissues used in the preparation of the cultures were resected by the same surgeons, and similar analyses were performed by the same researchers. The researchers who examined the results were blind to the drugs and dosages utilized in each group.

\section{Inclusion criteria and preparation of cell cultures from human primary intervertebral disc tissue}

The study included 16 volunteers admitted to the neurosurgery department of Namik Kemal University School of Medicine after spinal trauma. After lumbar magnetic resonance image evaluation, the intervertebral disc tissues of the patients who had posterior transpedicular stabilization, lumbar laminectomy, and discectomy as surgical modalities were included in the study.

Patients suffering from any liver disease with a risk of bleeding or any other disease that may increase the risk of serious bleeding in solid organs were excluded from the study. To prevent errors that may arise from drug interactions, patients using drugs such as warfarin, heparin, dabigatran, or apixaban were excluded from the study. Patients administered heparin to keep the venous/arterial line open were also excluded from the study. The following patients were also excluded from the study: those with moderate to severe renal insufficiency and patients using non-steroidal anti-inflammatory drugs, acetylsalicylic acid, blood clotting-inhibiting drugs, or azole antifungal agents, such as ketoconazole, itraconazole, voriconazole, and posaconazole, within the last 3 weeks. In addition, patients with acquired immune deficiency syndrome and those using human immunodeficiency virus protease inhibitors, such as ritonavir, were excluded from the study.

Of the tissues included in the study $(n=16)$, tissues of patients using erythromycin $(n=1)$, clarithromycin $(n=1)$, fluconazole $(n=1)$, enoxaparin $(n=2)$, clopidogrel $(n=1)$, or vitamin $\mathrm{K}$ antagonists, such as warfarin $(n=3)$ and naproxen $(n=1)$, within the last 3 weeks were excluded from the study. The remaining 6 patient tissues were used in the preparation of the primary cell culture.

The age range of the patients who met the inclusion criteria was 27-39 years. Patients with 
traumatic disc herniation ( $n=6$, mean age: 33 \pm 5.32 years) underwent discectomy and instrumentation using the posterolateral fixation technique. The tissues were then transferred to the laboratory at $4^{\circ} \mathrm{C}$.

Cell cultures containing intact human intervertebral disc tissue were prepared in accordance with the protocol [9-14]. The tissue samples, which were irrigated with $0.9 \%$ isotonic sodium chloride solution in a laminar flow cabinet (Air Flow-NUVE/NF-800 R, Ankara, Turkey), were then washed three times consecutively with phosphate buffered saline and clarified from the red blood cells. The tissues were first degraded mechanically using a rongeur and then treated with $0.375 \mu \mathrm{l}$ of Clostridium histolyticum (collagenase type II enzyme) (Invitrogen Corp., Sigma Chemical, St. Louis, MO) and Hanks' balanced salt solution (HBSS-1X, 14025 , Gibco) to perform the enzymatic degradation. This cell suspension was incubated overnight in an incubator (NUVE, 06750, Ankara, Turkey) with $5 \% \mathrm{CO}_{2}$ at $37^{\circ} \mathrm{C}$. The samples were subsequently centrifuged three times consecutively at $1,100 \mathrm{rpm}$ and $4^{\circ} \mathrm{C}$. The supernatants in the tubes were discarded. The cell pellets were re-suspended with a freshly prepared culture medium containing Dulbecco's modified Eagle's medium (DMEM; Cat\#41965062; Gibco) supplemented with $1 \%$ penicillin-streptomycin and $10 \%$ fetal bovine serum (FBS; Gibco). The cells were stained with trypan blue and counted using an inverted light microscope (magnification, 10x).

The counted cells were plated at $1.6 \times 10^{4}$ cells per well in 96-well plates, $1.1 \times 10^{4}$ cells per well in 24-well plates, and $2.8 \times 10^{6}$ cells per dish in petri dishes $(100 \mathrm{~mm})$ for 3-(4,5-dimethylthiazol-2-yl)-2,5-diphenyltetrazolium bromide (MTT) viability and proliferation. Toxicity assays, inverted light microscopy, acridine orange (AO), and propidium iodide $(\mathrm{PI})(\mathrm{AO} / \mathrm{PI})$ assays, and histopathological evaluations were conducted. After cell incubation overnight, rivaroxaban was added to the cell cultures that had become confluent and adhered to the cell culture plates or dishes.

\section{Rivaroxaban treatment of the primary cell cultures}

Based on EMEA 2008 [20], the inhibition-causing dose $\left(\mathrm{IC}_{50}\right)$ for rivaroxaban $(20 \mathrm{mg}$ ) (Xarelto Bayer Turk Kimya Sanayi) was $2.1 \mathrm{nM}$. Therefore, rivaroxaban was dissolved in dimethyl sulfoxide (DMSO) to obtain a $0.013 \mu \mathrm{g} / \mathrm{ml}$ stock solution. From this obtained stock solution, rivaroxaban in a final concentration of $4.66 \mu \mathrm{l} / \mathrm{ml}$ was diluted with culture medium and administered to the cultures of the experimental groups. Non-drugtreated cultures were used as the control group. Analyses of the non-drug-treated group and rivar- oxaban-treated groups were performed on days 10 and 20.

\section{Analyses}

Cell surface morphology and viability were examined using an inverted light microscope. Microscopical evaluations were performed under $4 \times$, $10 \times, 20 x$, and $40 x$ magnifications at $0 \mathrm{~h}$ and on days 10 and 20 . In addition, membrane permeability tests were conducted using a fluorescent microscope and nucleic acid binding dyes (AO and $\mathrm{PI})$ under similar magnifications. Based on the principle [9-14] that live cells produce green fluorescence and dead cells produce red fluorescence, the results of the analyses were evaluated.

The cell viability tests were conducted using a commercial MTT kit (Vybrant MTT Cell Proliferation Assay, Cat. No. V-13154; Thermo Fisher Scientific, Waltham, MA, USA). The MTT assay measures color changes, which occur due to the production of formazan by the cells undergoing proliferation, using tetrazolium with increasing dehydrogenase enzyme activity [21]. The quantity of formazan was measured by recording the changes in absorbance at $570 \mathrm{~nm}$ using a plate reading spectrophotometer. The viability of the cells in the control group samples, to which rivaroxaban was not initially applied, was designated as $100 \%$. The proliferation and the inhibition of the proliferation were calculated using the following formulas, respectively: Test OD/Control ODX100 and 1-Test $O D /$ Control OD. The data were recorded for statistical analysis [9-14].

The total amount of RNA was extracted from cultured primary human intact intervertebral disc cells using the PureLink RNA mini kit. The quantity of RNA obtained from each sample was measured using a UV spectrophotometer. To obtain cDNA, 50 ng RNA was reverse transcribed using a high-capacity CDNA reverse transcription kit and a thermal cycler [22]. All genes were amplified using TaqMan Gene Expression assays for CHAD (Cat\#4331182, Hs00154382_m1), an endogenous control (ACT $\beta$ ) (Cat\#4331182, Hs03023943 g1); COMP (Cat\#4331182, Hs00164359_m1); IL-1B (Cat\#4331182, Hs01555410_m1); MMP13 (Cat\#4331182, Hs00942583_g1); MMP-19 (Cat\#4331182, Hs00418247_g1); and MMP-7 (Cat\#4331182, Hs01042796_m1). qPCR was performed on an Applied Biosystems 7300/7500 real-time polymerase chain reaction (PCR) system (Thermo Fisher Scientific, Inc.). The thermocycling conditions were as follows: $2 \mathrm{~min}$ at $50^{\circ} \mathrm{C}, 10 \mathrm{~min}$ at $95^{\circ} \mathrm{C}, 15 \mathrm{~s}$ at $95^{\circ} \mathrm{C}$, and $1 \mathrm{~min}$ at $60^{\circ} \mathrm{C}$ for 40 cycles. Following the RT-qPCR experiment, the relative quantity (RQ) values of each sample were obtained using the 7500 Fast-SDS program V.2.3 (Thermo Fisher Scientific, Inc.). ACT $\beta$ was utilized 
to normalize the target gene expression. To obtain the comparative results, reference (calibrator) samples were used, and RQ values were calculated using the $2^{-\Delta \Delta_{\mathrm{ca}}}$ method $[10,11]$.

\section{Statistical analysis}

The statistical analysis were performed using SPSS (version 18.0) software, and the data were evaluated at the 95\% confidence interval. Descriptive statistics were presented as the mean \pm standard deviation. An analysis of variance test was performed to determine whether there were significant differences in the mean across the groups. When differences across groups were observed, Tukey's honestly significant difference post-hoc test was used for multiple pairwise comparisons. The $\alpha$ significance value was $<0.05$.

\section{Results}

Cell morphology, viability, and proliferation remained unchanged in AF/NPC cultures after rivaroxaban application according to inverted microscopy, AO/PI staining, and MTT analysis

Morphological evaluation of AF/NPC cultures demonstrated that the application of rivaroxaban for 10 to 20 days did not affect cell morphology or proliferation. When inverted microscopy images were examined, the number of cells and the amount of proliferation were similar in both experimental and control groups. Therefore, ECM formation was observed to be weaker in the group treated with rivaroxaban (Figures $1 \mathrm{~A}-\mathrm{E})$. AO/PI staining showed that cells from both experimental and control groups continued to proliferate within 10-20 days, and no apoptotic cell death was observed as a result of rivaroxaban administration (Figures $1 \mathrm{~F}-\mathrm{J}$ ).

Similarly, with AO/PI staining results, when cell viability was evaluated using the MTT analysis, it was confirmed that rivaroxaban at the administered dose did not suppress cell viability or proliferation (Figure 2). The data obtained from the MTT analysis were statistically significant $(p<0.05)$.

\section{Gene expression profiles were changed due to rivaroxaban administration}

To evaluate the gene expression of CHAD, COMP, IL-1B, MMP-13, MMP-19, and MMP-7 genes, pretestcultureswithoutrivaroxaban application (day0) were used as reference samples. The gene expression level in these AF/NPC cultures was designated as $100 \%(R Q=1)$. The expression levels of CHAD, COMP, IL-1B, MMP-13, MMP-19, and MMP-7 genes on days 10 and 20 were determined as a fold increase or decrease compared with the control group on the same days.
Accordingly, the expression of the CHAD, IL-1B, MMP-13, and MMP-7 genes remained unchanged on day 10, while the COMP expression decreased 0.4-fold and MMP-19 expression increased 0.4fold. On day 20, COMP and IL-1B were expressed at levels similar to the control group. However, CHAD expression was 3.3-fold higher, MMP-13 expression was 36.3-fold higher, MMP-19 expression was 0.7-fold higher, and MMP-7 expression was 4.5-fold higher compared with the control (Figure 3). Rivaroxaban application versus expression of genetic markers (CHAD, COMP, IL-1B, MMP-13, MMP-19, and MMP-7) and duration of application were statistically evaluated. The change in gene expression was statistically significant $(p<0.05)$ (Table I).

\section{Discussion}

A complex set of anabolic and catabolic reactions provides continuity to the matrix structure of intervertebral disc tissue. Matrix-degrading enzymes, such as COMP, a member of the disintegrin and metalloprotease with thrombospondin motifs (ADAMTS) family, are thought to play a key role during these reactions. MMPs and COMP are known to cause a decrease in the expression of both aggrecan and type II collagen genes and in the overall synthesis of aggrecan and collagen as a result of the induction of proinflammatory cytokines, which are involved in the matrix construction and destruction cycle in the nucleus pulposus [23].

The absence of lymphatic vessels in the intervertebral disc tissue leads to an increase in the density of matrix destructive factors and their effects. In addition to this adverse condition, it is known that many drugs that are administered orally or parenterally accumulate in the synovial fluid [9-14, 24, 25]. Because the outer layer of the synovial tissue is thicker, the drugs and/or nutrients diffuse from the hyaluronan and synovial tissues to the body fluids. They then pass through pores in the hyaline membranes located at the intervertebral disc space and reach the intervertebral disc tissue cells [9-14].

The aim of the present study was to investigate the effects of rivaroxaban, a novel oral Xa inhibitor, on primary cell cultures isolated from intact human intervertebral disc tissue. Rivaroxaban is used for the prevention of the risk of early in-hospital death due to acute stroke [4] and for recurrent symptomatic or fatal venous thromboembolism in patients with acute pulmonary embolism. Rivaroxaban is frequently prescribed by many clinicians, including neurosurgeons, as it is markedly effective for a long time, and it does not require a constant dosing regimen or routine laboratory monitoring [26-29]. In addition to the advantages of the drug, however, it has serious side effects. 


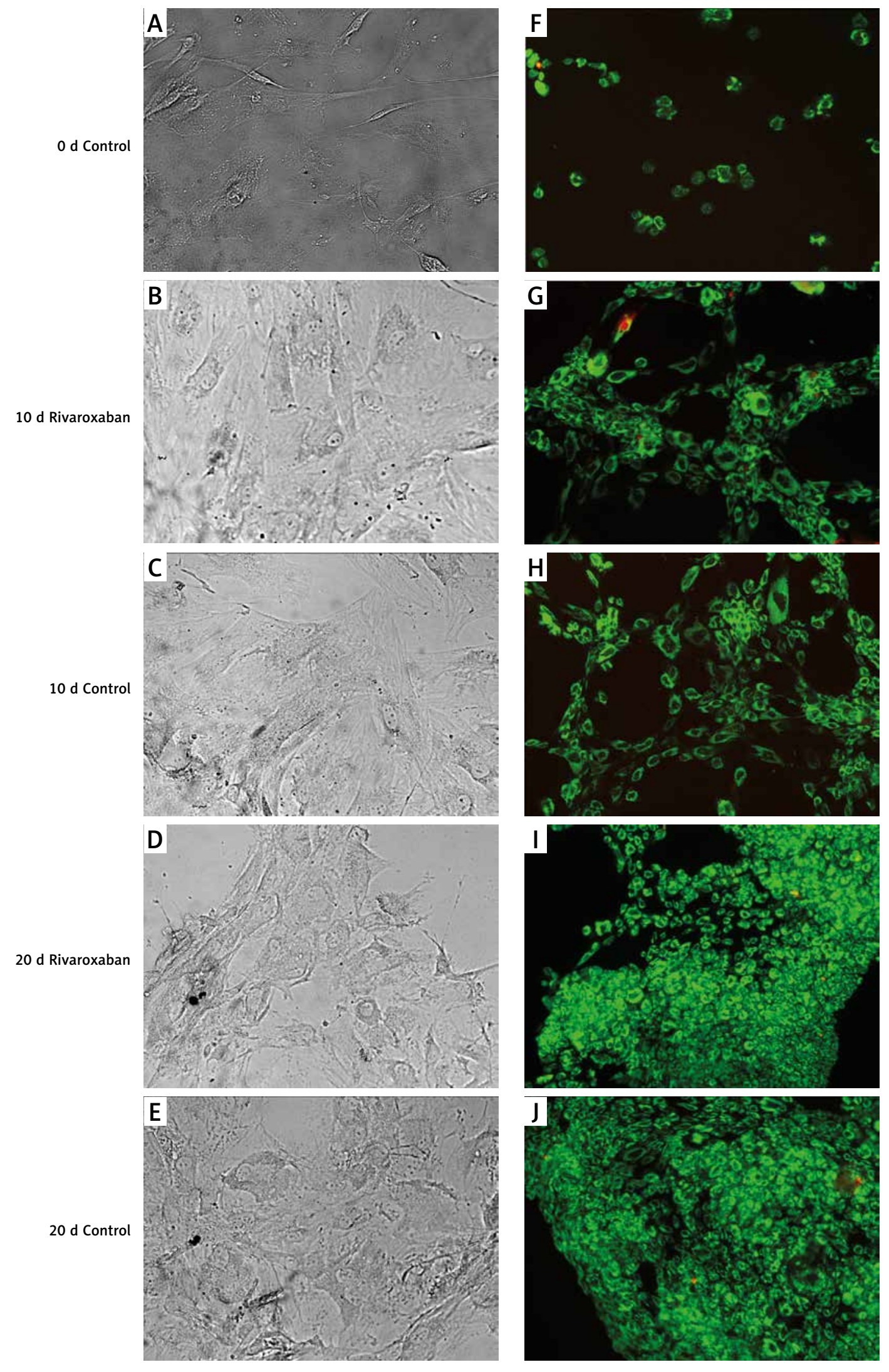

Figure 1. Microscopy of nucleus pulposus and annulus fibrosus cell cultures: A-E - represent inverted light microscopy (first column) under 20x magnification, $\mathbf{F}-\mathbf{J}-$ represent fluorescent microscopy after AO/PI staining (second column) under $10 \times$ magnification. $\mathbf{A}$ and $\mathbf{F}$ are micrographs of control group cells after first passage incubated for 0 days. B, G, D, and I are rivaroxaban-treated cultures for 10 and 20 days, respectively. C, H, E, and J are micrographs of non-treated control group cultures at 10 and 20 days, respectively 


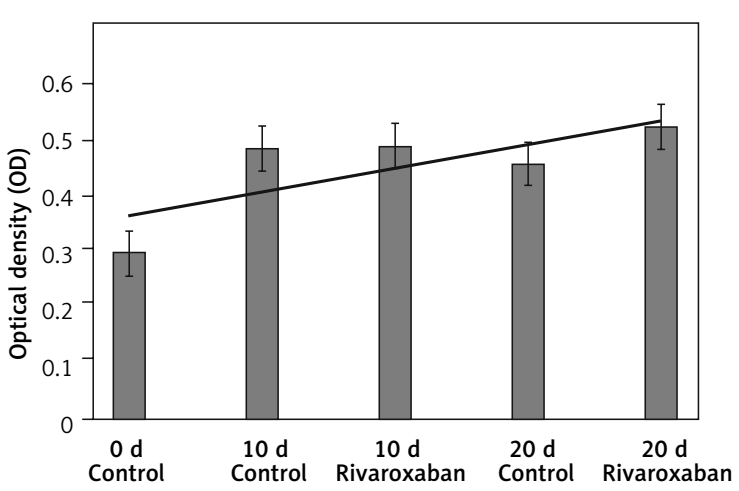

Figure 2. Results of the MTT analysis performed through the spectrophotometric methodology at $570 \mathrm{~nm}$

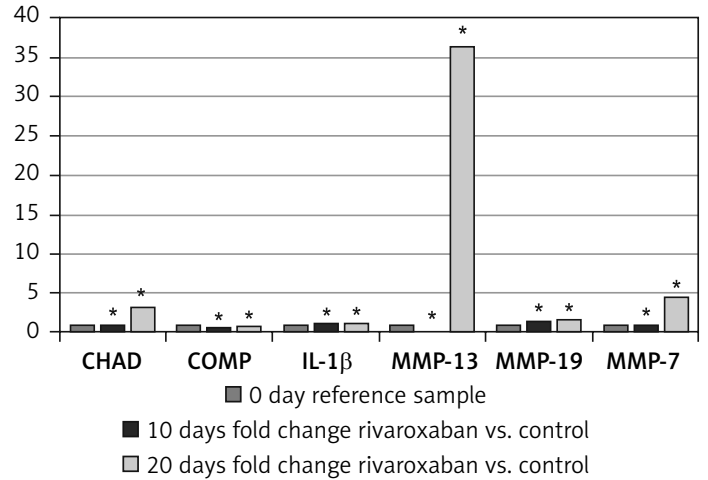

Figure 3. Fold changes in the expression of $C H A D$, COMP, IL-1 1 , MMP-13, MMP-19, and MMP-7 genes on days 10 and 20 compared with the control group on the same days

Table I. Relationship between incubation period and gene expression in the rivaroxaban-treated samples

\begin{tabular}{|lllcc|}
\hline Source & Adj. SS & Adj. MS & F-value & $P$-value* \\
\hline Rivaroxaban & 22.850 & 22.8500 & 21.54 & $<0.001$ \\
\hline Markers & 95.889 & 31.9631 & 30.14 & $<0.001$ \\
\hline Times & 35.971 & 17.9855 & 16.96 & $<0.001$ \\
\hline Rivaroxaban vs. markers & 24.041 & 8.0138 & 7.56 & $<0.001$ \\
\hline Markers vs. times & 51.210 & 8.5349 & 8.05 & $<0.001$ \\
\hline
\end{tabular}

Adj. SS - adjusted sum of squares; Adj. MS - adjusted mean square; *analysis of variance test (ANOVA, $p<0.05$ ).

Previous studies suggested that the use of rivaroxaban might exacerbate intracranial hemorrhage [30] in patients with mild traumatic brain injuries and thereby might cause serious and fatal complications, such as intracerebral hematoma, intracranial hemorrhage, and nontraumatic spinal subdural hematoma [31-34].

However, no previous study has focused on the positive or negative effects of this pharmacological agent on intervertebral disc tissue. Therefore, the findings of the present study would contribute to the literature.

Dhar et al. [35] investigated the effects of rivaroxaban, which is a prodrug and direct $\mathrm{Xa}$ inhibitor, and dabigatran, which is a non-prodrug and direct factor Ila inhibitor, on $\alpha$-smooth muscle actin ( $\alpha S M A)$ using hepatic stellate cells-LX2 cells. They concluded that the early inhibition of coagulation using rivaroxaban, which is an FXa inhibitor, significantly reduced TAA-induced murine liver fibrosis [35].

Hashikata et al. [36] investigated the effects of rivaroxaban on cell migration, proliferation, and differentiation in cardiac fibroblast cells, which play a central role in cardiac fibrosis. They reported that rivaroxaban inhibited Ang II-induced cell proliferation and migration and that it blocked various inflammatory signal pathways [36].

Gigi et al. [20] investigated the direct effects of rivaroxaban on bone biology using the human fe- male osteoblastic cell line SaOS2. They treated the cells with different concentrations of rivaroxaban for $24 \mathrm{~h}$ and conducted analyses to determine DNA synthesis and creatine kinase- and alkaline phosphatase-specific activities. They then tested bone mineralization on day 21 [20]. They reported that rivaroxaban $(0.01-50 \mu \mathrm{g} / \mathrm{ml})$ inhibited up to $60 \%$ of the DNA synthesis of the cells and that it inhibited dose-dependent creatine kinase-specific activity when it was applied in the same concentrations. They also reported that rivaroxaban inhibited dose-dependent alkaline phosphatase-specific activity up to $30 \%$ [20]. They concluded that rivaroxaban inhibited the first stage of bone formation but did not affect later stages [20].

Commercial cell lines or animal tissues have been used in some studies [20, 35, 36]. However, commercial cell lines contain a single type of cell and do not have original phenotypic and genotypic characteristics. Therefore, the results obtained in previous studies using a commercial cell line may be misleading [9-14, 24, 25]. It is well known that human and animal tissues differ in sensitivity. Thus, the results obtained from studies on live mammals may differ from those obtained in human studies; therefore, such results may be misleading [24, 25, 37, 38].

In the present study, we did not use animal tissues or commercial cell lines. Instead, we prepared human primary cell cultures from intact and 
healthy human intervertebral disc tissue, and we evaluated the effects of rivaroxaban on these cultures. Our findings contribute to the value of the present study for the relevant literature.

Our results showed that the expression of CHAD, IL-1B, MMP-13, and MMP-7 genes remained unchanged on day 10 , whereas COMP expression decreased and MMP-19 expression increased. On day 20, COMP and IL-1B were expressed at levels similar to the control group. The expression levels of CHAD, MMP-13, MMP-19, and MMP-7 were higher compared with the control $(p<0.05)$. The results which were obtained following the statistical evaluations of both proliferation and gene expression were markedly significant $(p<0.05)$.

Disc degeneration is defined as progressive changes that affect the spine and which develop due to the natural aging process and multiple other factors [39]. The leading causes of the degeneration of intervertebral disc tissue are degenerative changes related to drug use [9-14, 24, 25]. MMPs are important proteolytic enzymes that are members of the endopeptidase family, which can degrade ECM components. The ECM plays a crucial role in maintaining cell viability, cell migration, and regulation of the interrelationships among cells [40]. In our study, it was determined that 10day rivaroxaban administration did not change MMP-13 and MMP-7 expression, but increased MMP-19. However, when the application period increased to 20 days, an increase was observed in the amount of all MMPs studied. No toxic effect of rivaroxaban was detected in our treated cultures, but it was observed that culture development was weaker than the control group, especially in terms of adhesion and ECM formation. With this finding, although it is not possible to claim that MMP increase alone will slow down the clinical recovery process, it shows that the duration of drug use should be carefully evaluated.

Several studies have reported that members of the MMP and ADAMTS families of enzymes are prone to damaging cartilage tissues and cartilage-like tissues [41]. It is well known that COMP is a member of the ADAMTS family [40]. It has been reported in the literature that inflammatory cytokines stimulate ADAMTS- 5 and increase COMP tissue damage more than MMPs do [42-44]. In our study, it was determined that rivaroxaban application decreased COMP expression on day 10, but the expression was normalized on day 20. In this context, it can be argued that in terms of COMP gene expression, AF/NP tissue can resist the application and will not cause any tissue damage over COMP increase. In addition to this increase, drugs accumulated in the tissues increase the effects of the matrix destructive factors on intervertebral disc tissues that are already aliphatic, thereby exacerbating catabolic effects. Thus, the catabol- ic process can be accelerated, and more intensive catabolic effects can be observed.

In addition to these findings, it was determined that the expression level of CHAD, an NP-specific marker, did not change on the $10^{\text {th }}$ day and increased on the $20^{\text {th }}$ day. These data indicate that rivaroxaban does not particularly affect NP cell proliferation. Similarly, the fact that IL-1b expression remained unchanged at days 10 and 20 suggests that the administration does not have a negative effect in terms of inflammation.

In conclusion, the results suggest that it is likely that recently released rivaroxaban can reduce matrix synthesis in disc tissue. Therefore, clinicians should not forget that rivaroxaban may suppress tissue healing due to drug-use duration. Although changes in a determined group of genes cannot always explain the prognosis alone, it should always be carefully evaluated considering genetic instability that may be caused by changes in gene expression level. Rivaroxaban does not have any acute toxic effects, but when the application time of rivaroxaban is kept to 20 days or more, increasing MMP expression can negatively affect the healing process.

\section{Conflict of interest}

The authors declare no conflict of interest.

\section{References}

1. Ofek F, Barchel D, Perets N, et al. International normalized ratio as a screening test for assessment of anticoagulant activity for patients treated with rivaroxaban or apixaban: a pilot study. Front Pharmacol 2019; 10: 1177.

2. Li X, Zuo C, Lu W, et al. Evaluation of remote pharmacist-led outpatient service for geriatric patients on rivaroxaban for nonvalvular atrial fibrillation during the COVID-19 pandemic. Front Pharmacol 2020; 11: 1275.

3. Hartmann S, Biliouris K, Lesko LJ, Nowak-Göttl U, Trame MN. Quantitative systems pharmacology model-based predictions of clinical endpoints to optimize Warfarin and Rivaroxaban anti-thrombosis therapy. Front Pharmacol 2020; 11: 1041.

4. Hart RG, Sharma M, Mundl H, et al. Rivaroxaban for stroke prevention after embolic stroke of undetermined source. N Engl I Med 2018; 378: 2191-201.

5. Hurtado-Navarro I, García-Sempere A, Rodríguez-Bernal C, Santa-Ana-Tellez Y, Peiró S, Sanfélix-Gimeno G. Estimating adherence based on prescription or dispensation information: impact on thresholds and outcomes. A real-world study with atrial fibrillation patients treated with oral anticoagulants in Spain. Front Pharmacol 2018; 9: 1353.

6. Naito T, Hayashi H, Kashiwada T, et al. Pulmonary embolism and deep vein thrombosis in eosinophilic granulomatosis with polyangiitis successfully treated with rivaroxaban. Respir Med Case Rep 2018; 25: 33-5.

7. Piotrowski R, Zaborska B, Pilichowska-Paszkiet E, Sikora-Frąc M, Baran J, Kułakowski P. RIVAroxaban TWICE daily for lysis of thrombus in the left atrial append- 
age in patients with non-valvular atrial fibrillation: the RIVA-TWICE study. Arch Med Sci 2019; 16: 289-96.

8. Koscielny J, Beyer-Westendorf J, von Heymann C, et al. Risk of bleeding and haemorrhagic complication with rivaroxaban: periprocedural management of haemostasis. Hamostaseologie 2012; 32: 287-93.

9. Caliskan T, Sirin DY, Karaarslan N, et al. Effects of etanercept, a tumor necrosis factor receptor fusion protein, on primary cell cultures prepared from intact human intervertebral disc tissue. Exp Ther Med 2019; 18: 69-76.

10. Karaarslan N, Yilmaz I, Sirin DY, et al. Pregabalin treatment for neuropathic pain may damage intervertebral disc tissue. Exp Ther Med 2018;16: 1259-65.

11. Kaya YE, Karaarslan N, Sirin DY, Ozbek H, Kaplan N, Yilmaz I. Investigation of the effects of methylphenidate, an amphetamine derivative, on intervertebral disc tissue cell cultures and matrix structures. Turk Neurosurg 2019; 29: 734-42

12. Akgun FS, Sirin DY, Yilmaz I, et al. Investigation of the effect of dipyrone on cells isolated from intervertebral disc tissue. Exp Ther Med 2019; 18: 216-24.

13. Kaplan N, Karaarslan N, Yilmaz I, et al. Are intervertebral disc tissue cells damaged when attempting to prevent thrombus formation using dabigatran, a new oral anticoagulant? Turk Neurosurg 2019; 29: 470-7.

14. Karaarslan N, Yilmaz I, Ozbek H, et al. Are specific gene expressions of extracellular matrix and nucleus pulposus affected by primary cell cultures prepared from intact or degenerative intervertebral disc tissues? Turk Neurosurg 2019; 29: 43-52.

15. Chen FH, Herndon ME, Patel N, Hecht JT, Tuan RS, Lawler J. Interaction of cartilage oligomeric matrix protein/ thrombospondin 5 with aggrecan. J Biol Chem 2007; 282: 24591-8.

16. Veras MA, McCann MR, Tenn NA, Séguin CA. Transcriptional profiling of the murine intervertebral disc and age-associated changes in the nucleus pulposus. Connect Tissue Res 2020; 61: 63-81.

17. Mao SH, Qian BP, Shi B, Zhu ZZ, Qiu Y. Quantitative evaluation of the relationship between COMP promoter methylation and the susceptibility and curve progres sion of adolescent idiopathic scoliosis. Eur Spine J 2018 27: 272-7.

18. Goode AP, Marshall SW, Kraus VB, et al. Association between serum and urine biomarkers and lumbar spine individual radiographic features: the Johnston County Osteoarthritis Project. Osteoarthritis Cartilage 2012; 20: 1286-93.

19. Kwon WK, Moon HJ, Kwon TH, Park YK, Kim JH. the role of hypoxia in angiogenesis and extracellular matrix regulation of intervertebral disc cells during inflammatory reactions. Neurosurgery 2017; 81: 867-75.

20. Gigi R, Salai M, Dolkart O, et al. The effects of direct factor Xa inhibitor (Rivaroxaban) on the human osteoblastic cell line SaOS2. Connect Tissue Res 2012; 53: 446-50.

21. Xu N, Wang L, Fu S, Jiang B. Resveratrol is cytotoxic and acts synergistically with NF- $\mathrm{BB}$ inhibition in osteosarcoma MG-63 cells. Arch Med Sci 2020; 17: 166-76.

22. Sirin DY, Karaarslan N. Evaluation of the effects of pregabalin on chondrocyte proliferation and CHAD, HIF-1 $\alpha$, and COL2A1 gene expression. Arch Med Sci 2018; 14: 1340-7.

23. Séguin CA, Pilliar RM, Roughley PJ, Kandel RA. Tumor necrosis factor-alpha modulates matrix production and catabolism in nucleus pulposus tissue. Spine 2005; 30: 1940-8.
24. Gumustas SA, Yilmaz I, Isyar M, et al. Assessing the negative impact of phenyl alkanoic acid derivative, a frequently prescribed drug for the suppression of pain and inflammation, on the differentiation and proliferation of chondrocytes. J Orthop Surg Res 2016; 11: 70.

25. Gumustas F, Yilmaz I, Sirin DY, et al. Chondrocyte proliferation, viability and differentiation is declined following administration of methylphenidate utilized for the treatment of attention-deficit/hyperactivity disorder. Hum Exp Toxicol 2017; 36: 981-92.

26. Chunhong L, Jin L, Qin Z, Guohui X. Anticoagulant effects of rivaroxaban after surgical fixation of spinal fracture. Pak J Pharm Sci 2018; 31: 1131-5.

27. Naito T, Hayashi H, Kashiwada T, et al. Pulmonary embolism and deep vein thrombosis in eosinophilic granulomatosis with polyangiitis successfully treated with rivaroxaban. Respir Med Case Rep 2018; 25: 33-5.

28. Wu CT, Chen B, Wang JW, Yen SH, Huang CC. Plasma $\mathrm{D}$-dimer is not useful in the prediction of deep vein thrombosis after total knee arthroplasty in patients using rivaroxaban for thromboprophylaxis. J Orthop Surg Res 2018; 13: 173.

29. Bhatnagar UB, Rezkalla J, Sethi P, Stys A. Successful resolution of a large left ventricular thrombus with rivaroxaban therapy after acute myocardial infarction. S D Med 2018; 71: 62-3.

30. Beynon C, Potzy A, Sakowitz OW, Unterberg AW. Rivaroxaban and intracranial haemorrhage after mild traumatic brain injury: a dangerous combination? Clin Neurol Neurosurg 2015; 136: 73-8.

31. Tao J, Bukanova EN, Akhtar S. Safety of 4-factor prothrombin complex concentrate (4F-PCC) for emergent reversal of factor Xa inhibitors. J Intensive Care 2018; 6: 34.

32. Wasserlauf G, Grandi SM, Filion KB, Eisenberg MJ. Meta-analysis of rivaroxaban and bleeding risk. Am J Cardiol 2013;112: 454-60.

33. Baschera D, Oberle J, Grubhofer F, Schmid SL. Perioperative use of anticoagulant and platelet-inhibiting medications for elective spine surgery: results of a nationwide survey. J Neurol Surg A Cent Eur Neurosurg 2018; 79: 398-407.

34. Kawabori M, Niiya Y, Iwasaki M, et al. Characteristics of symptomatic intracerebral hemorrhage in patient receiving direct oral anticoagulants: comparison with warfarin. J Stroke Cerebrovasc Dis 2018; 27: 1338-42.

35. Dhar A, Sadiq F, Anstee QM, Levene AP, Goldin RD, Thursz MR. Thrombin and factor Xa link the coagulation system with liver fibrosis. BMC Gastroenterol 2018; 18: 60.

36. Hashikata T, Yamaoka-Tojo M, Namba S, et al. Rivaroxaban Inhibits Angiotensin II-induced activation in cultured mouse cardiac fibroblasts through the modulation of NF- B pathway. Int Heart J 2015; 56: 544-50.

37. Yilmaz I, Karaarslan N, Ozbek H. Practical performance of hippocampal tissue resection in rats in pharmacomolecular research. Turk Neurosurg 2021; 31: 112-8.

38. Yilmaz I, Karaarslan N, Ozbek H. Pharmaco-molecular assessment of the effects of anandamide and its antagonists on hippocampal tissue in Wistar albino rats. Eur Rev Med Pharmacol Sci 2020; 24: 11871-82.

39. Hu X, Cao D, Wang Z. Metformin alleviates intervertebral disc degeneration by upregulating MMP-1 expression via the KDM6A/SOX9/miR-202-3p/MMP-1 signaling pathway. Arch Med Sci 2021. doi:10.5114/ aoms/117427.

40. Liang Y, Fu Y, Qi R, et al. Cartilage oligomeric matrix protein is a natural inhibitor of thrombin. Blood 2015; 126 : 905-14. 
41. Min N, Ma J, Shi L, et al. miR-223 promotes cartilage differentiation of bone marrow-derived mesenchymal stem cells and protects against osteoarthritis by suppressing NLRP-3 expression. Arch Med Sci 2020. doi:10.5114/aoms.2020.100640.

42. Yang CY, Chanalaris A, Troeberg L. ADAMTS and ADAM metalloproteinases in osteoarthritis: looking beyond the 'usual suspects'. Osteoarthritis Cartilage 2017; 25: 1000-9.

43. Cal S, Obaya AJ, Llamazares M, Garabaya C, Quesada V, Lopez-Otin C. Cloning, expression analysis, and structural characterization of seven novel human ADAMTSs, a family of metalloproteinases with disintegrin and thrombospondin-1 domains. Gene 2002; 283: 49-62.

44. Roberts S, Caterson B, Menage J, Evans EH, Jaffray DC, Eisenstein SM. Matrix metalloproteinases and aggrecanase: their role in disorders of the human intervertebral disc. Spine 2000; 25: 3005-13. 